\title{
SOCIOLOGÍA Y ALIMENTACIÓN
}

\author{
CECILIA DÍAZ MÉNDEZ y CRISTÓBAL GÓMEZ BENITO
}

Universidad de Oviedo y UNED

\section{PALABRAS CLAVE ADICIONALES}

Cocina, Gusto, Dieta y Nutrición.

\author{
ADDITIONAL KEYWORDS \\ Cuisine, Taste, Diet and Nutrition.
}

RESUMEN. En este trabajo, se exponen las relaciones entre la sociología y el fenómeno alimentario. En primer lugar, se apuntan algunas de las razones que podrían explicar el retraso con que la sociología se ha ocupado de forma sistemática de este ámbito de la vida social y las condiciones que han despertado el interés reciente de esta disciplina por el fenómeno alimentario. En segundo lugar, se presenta un esquema de la atención prestada por los clásicos de la sociología a la alimentación, de modo que se pueda contrastar la naturaleza de esta atención con el análisis sociológico actual. En tercer lugar, se presenta un esquema de las principales orientaciones teóricas que han marcado la sociología (y la antropología) de la alimentación, para terminar con una propuesta de definición del campo de la sociología de la alimentación a partir de la consideración de los contenidos de algunos textos actuales de este campo de estudio.

ABSTRACT. This work presents briefly the relationship between sociology and food matters. In the first place, we point out some of the reasons by which we might explain why sociology has only lately started to systematically tackle this aspect of social life and we also point out what conditions have brought about this recent interest of this discipline in food. Secondly, we include an outline of the attention paid to food by some of the most important sociology scholars. Then we present an outline of the main theories underlying food sociology (and anthropology) and we end up with a proposal to define the field of food sociology, starting from the analysis of the content of some of the most important current works on the sociology of food.

E-mail: cecilia@uniovi.es_ａmez@poli.uned.es

Revista Internacional de Sociología (RIS)

Tercera Época, Nº40, Enero-Abril, 2005, pp. 21-46. 


\section{INTRODUCCIÓN}

Es bien sabido que hasta hace pocos años la sociología apenas había prestado atención a la alimentación. La comida y los comportamientos asociados a ella no habían sido considerados como temas de estudio relevantes por los sociólogos. Diversos autores han resaltado el retraso con que la sociología ha abordado, de forma sistemática y central, el fenómeno alimentario (Mennell, Murcott y Otterloo, 1992; McIntosh, 1996, Poulain, 2002). Mennell, Murcott y Otterloo (1992:1) apuntan varias razones sobre este desinterés: en primer lugar, sugieren que el retraso se debe a que la alimentación responde a una necesidad biológica ${ }^{1}$; en segundo lugar, apuntan hacia la cotidianeidad de la alimentación en la vida social, presentándola con frecuencia como un hecho dado; en tercer lugar, no ignoran que la sociología se ha centrado, en sus primeros desarrollos, en la sociedad industrial, restando curiosidad a las cuestiones del consumo y a aquellas áreas de la vida social no productivas (la esfera doméstica en particular); en último lugar, nos recuerdan que el hambre ha dejado de ser hace bastantes años un problema social prioritario en las sociedades occidentales desarrolladas.

Sin duda, la parte biológica del acto de comer tiene que ver con este distanciamiento entre la sociología y la alimentación, y ello a pesar de los debates entre lo biológico y cultural que han caracterizado una buena parte de los avances de esta ciencia ${ }^{2}$. Por el contrario, han sido sobre todo los análisis antropológicos sobre la comida en diferentes sociedades los responsables de la consideración cultural y social de la alimentación. De manera quizás excesiva aunque convincente, algunos antropólogos han afirmado que la propia utilización del fuego por parte de los pueblos primitivos obligó a manipular culturalmente la comida. El

\footnotetext{
${ }^{1}$ En este sentido conviene recordar que en la definición de hecho social, Durkheim afirma que "Todos los individuos beben, duermen, comen y razonan, y la sociedad tiene el mayor interés en que esas funciones se lleven a cabo regularmente. Así pues, si estos hechos fuesen sociales, la sociología no tendría un objeto de estudio que le fuese propio y su ámbito se confundiría con el de la biología y el de la psicología" (1998:56). Pero más adelante, Durkheim considera que algunas prácticas relacionadas con la alimentación, como el intercambio de alimentos entre grupos o las prohibiciones alimentarias en algunas sociedades, son "modos de hacer que pueden ejercer una coerción social sobre el individuo", y, en ese caso, pueden ser consideradas como hechos sociales (1998:68).

${ }^{2}$ Hacemos aquí referencia a los debates sobre las bases de la sociedad, que pueden ir desde las aproximaciones de la sociobiología y sus críticos hasta los análisis teóricos de la Cultura y la explicación del proceso de socialización. Cualquier manual de sociología se hace eco de esta controversia. Un libro de referencia para conocer la superación de este debate es el de Lamo de Espinosa (1996).
} 
paso de lo crudo a lo cocinado ${ }^{3}$ mencionado por Levi-Strauss (1968), constituye para este autor el proceso determinante de la emergencia de la humanidad. Este simple hecho hace de la alimentación un comportamiento cultural. En general, las revisiones estructuralistas sobre la alimentación (más allá de Levi-Strauss) permiten hablar de la comida no como un asunto exclusivamente nutritivo, sino como algo inherente a las propias sociedades. Las prácticas alimentarias no sólo son comportamientos o hábitos, sino también, y sobre todo, prácticas sociales con una clara dimensión imaginaria, simbólica y social.

El papel decisivo de la alimentación en la evolución humana ha sido resaltado también por los sociólogos. Fischler indica que "la alimentación es una función biológica vital y al mismo tiempo una función social esencial. (...). Sus facetas innumerables se ordenan según dos dimensiones por lo menos. La primera se extiende de lo biológico a lo cultural, de la función nutritiva a la función simbólica. La segunda, de lo individual a lo colectivo, de lo psicológico a lo social. El hombre biológico y el hombre social, la fisiología y lo imaginario, están estrecha y misteriosamente mezclados en el acto alimenticio" (1995:14-15). Para comprender completamente el fenómeno de la alimentación hay que considerar, como dice Fischler, al "hombre biológico y al hombre social o cultural", de la misma manera que para comprender al "comensal moderno" hay que interrogarse sobre el "comensal inmemorial" y entre los dos está el "comensal eterno" (1995:14). Así pues, las prácticas alimentarias no sólo son comportamientos o hábitos, pues en esto los humanos no se diferencian del resto de las especies, sino también, y sobre todo, prácticas sociales, comportando por ello una dimensión imaginaria, simbólica y social.

Acercándonos a la actualidad es probable que ningún analista pueda ignorar el componente social de la comida. La constatación de los cambios sociales en el comportamiento alimentario a lo largo de la modernización de la sociedad ha puesto de manifiesto la creación de una cultura alimentaria paralela a la construcción del mundo moderno. Como veremos en algunos de los trabajos incluidos en este mismo número monográfico de la RIS, los autores que exploran el comportamiento alimentario desde diferentes perspectivas ofrecen un tratamiento analítico de la comida que no deja dudas sobre su carácter social. No hay que ignorar tampoco que este reciente acercamiento social a la alimentación se sustenta en el hecho de que, en cierto modo, lo biológico ha despertado de nuevo. Aunque la alimentación

\footnotetext{
${ }^{3}$ A veces se traduce el título del libro de Levi-Strauss por "Lo crudo y lo cocido". Creemos que es más apropiado hablar de cocinado y no de cocido, pues introduce con mayor claridad la intervención humana en la manipulación del alimento, que es la intención última del autor. Sobre el papel de la cocción de los alimentos (la primera expresión de la cocina) en la evolución humana puede verse también la obra de Johan Goudsblom (1992).
} 
no se entienda como un comportamiento estrictamente asociado a la parte biológica del individuo, las investigaciones sobre el genoma humano y los descubrimientos reciente sobre el origen de la especie humana ${ }^{4}$ devuelven importancia a aquello que siempre había estado asociado (aunque no siempre de modo correcto) a lo estrictamente orgánico e inquebrantable de la naturaleza humana. Quizás de forma más general la revitalización de la sociología de la cultura y la creciente apertura de la sociología hacia otros ámbitos (la antropología, la historia y la biología en particular) han favorecido también una atención mayor del fenómeno alimentario.

Otro de los aspectos que han alejado a los sociólogos del estudio de la comida ha sido la cotidianeidad del comportamiento alimentario. Siendo algo tan rutinario y constante, parece que forma parte de lo conocido, no de lo ignorado. Esto no sólo ha sucedido con la comida. Existen áreas concretas de la vida de las personas que han ocupado siempre un segundo lugar en los estudios sociológicos. El consumo mismo ha pasado a ser central en los análisis sociológicos cuando se ha vislumbrado como un elemento decisivo para el mantenimiento del sistema económico y cuando su relación con la producción se ha vuelto conflictiva. La comida, desde la perspectiva productiva, sólo es importante en la medida en que responde a las expectativas de la producción, y de ahí que haya aparecido en la escena sociológica (salvo dentro de determinadas subdisciplinas, como la sociología rural) a través del aspecto más claramente económico, el consumo alimentario.

Pero los temas relacionados con la alimentación han quedado marginados también por formar parte de la esfera privada de los individuos, y por ello mismo revitalizado ahora. Muy recientemente, la sociología ha fijado su mirada hacia lo que sucede en la esfera doméstica, y lo ha hecho a través de los análisis de género dentro del hogar. El análisis del trabajo doméstico, básicamente desde una perspectiva feminista, ha mostrado las actividades realizadas por las mujeres en los hogares, actividades antes invisibles fundamentalmente por formar parte de la economía informal. Entre estas actividades ignoradas aparece también la alimentación. Lo doméstico se ha visto particularmente favorecido en esta nueva visión del mundo, poniendo de manifiesto el peso de las mujeres en el funcionamiento de la vida social.

Por otra parte, ha ido en aumento el interés por la alimentación al despertarse, en varios sentidos, una cierta conciencia social sobre los problemas nutricionales en el mundo. El hambre mundial no pasa desapercibida. El premio Nobel Amartya Sen ha puesto de manifiesto los problemas de poder asociados a las hambrunas, en su libro Pobreza y hambre (1981), y demuestra que el hambre no es el resultado

\footnotetext{
${ }^{4}$ Nos referimos aquí a los hallazgos de Atapuerca, que han situado en una nueva dimensión de análisis el origen de la especie humana.
} 
de la escasez de alimentos disponibles, sino de la existencia de desigualdades en el acceso a la comida. Pero además, en los países industrializados aparecen desordenes alimentarios sorprendentes, como la bulimia y la anorexia, que parecen inexplicables en una sociedad caracterizada por la suficiencia alimentaria e incluso por la sobreabundancia de alimentos. A esto hay que añadir el aumento de las preocupaciones surgidas de la mano de los riesgos alimentarios (en particular los riesgos para la salud), que visualizan, además, los efectos ecológicos de la producción agraria en un momento en el que los valores sociales resaltan la protección del medio ambiente.

Sea como fuere, el caso es que existe un retraso en el análisis sociológico de los comportamientos alimentarios, de modo que hasta los años ochenta no se registran intentos sistemáticos de construir una Sociología de la Alimentación. El planteamiento inicial sobre si la alimentación es o no un hecho social ha dejado hoy de ser un tema de debate entre aquellos analistas que se han atrevido a considerar que existen suficientes soportes teóricos y empíricos para hablar de una Sociología de la Alimentación. Este es el caso de los sociólogos franceses y del ámbito anglosajón (y también, aunque en menor medida, de Italia), en los que se han desarrollado unas tradiciones potentes de estudios alimentarios, conectadas en muchos casos con la antropología y con la historia. En España, aún se está muy lejos de esta situación a pesar del incremento en los últimos quince años ${ }^{5}$ de los estudios sociológicos, antropológicos e históricos sobre la alimentación.

Los trabajos españoles sobre comportamiento alimentario realizados por sociólogos se encuentran adscritos a dos áreas: la sociología del consumo y la sociología rural, con un escaso vínculo entre ellas ${ }^{6}$. En Francia, Poulain (2002) ha aglutinado la diversidad de estudios sociales sobre este tema en su libro Sociologies de l'alimentation. En el ámbito anglosajón Mennell, Murcott y van Otterloo acuñan el término agrupando un gran número de trabajos empíricos y teóricos en un monográfico de Current Sociology, The sociology of food: eating, diet and culture (1992). En España, las tentativas de presentar un marco sobre la investigación del comportamiento alimentario se han producido desde el ámbito de la antropología a partir, sobre todo, de Gracia Arnáiz (1997 y 2002) quien presenta una agenda sobre este tema.

\footnotetext{
${ }^{5}$ Sobre el contexto del desarrollo de la sociología de la alimentación en España puede verse Díaz Méndez y Gómez Benito (2002).

${ }^{6}$ Pueden verse estas divisiones y la posición que ocupan en ellos los estudios sobre alimentación, en los grupos de trabajo del reciente VIII Congreso Español de Sociología (septiembre, 2004) http://www.fes-web.org
} 


\section{LA ALIMENTACIÓN EN LA TRADICIÓN SOCIOLÓGICA Y ANTROPOLÓGICA?}

En los últimos años se han realizado diferentes revisiones desde las Ciencias Sociales con el fin de concretar los orígenes de una Sociología de la Alimentación que parece cobrar forma en el ámbito académico internacional. Si bien, las clasificaciones de los autores considerados clásicos son motivos de divergencias. Aunque se discrepa sobre la adecuación de enmarcar a un determinado autor en una corriente teórica concreta, en la mayor parte de los trabajos, tanto si se trata de una revisión hecha por un antropólogo, como si la realiza un sociólogo, se presenta un elenco de autores que desde las Ciencias Sociales son utilizados como referentes teóricos de manera consistente para la comprensión de los resultados empíricos sobre el comportamiento alimentario. Esta coincidencia justifica, en nuestra opinión, la consulta obligada a los clásicos para una inmersión en el ámbito alimentario desde una perspectiva social ${ }^{8}$. La mayoría de los autores que se reseñan como clásicos no estudian el comportamiento alimentario en las sociedades modernas, pero no se pueden entender los trabajos de las últimas décadas sin estos soportes teóricos9.

Los primeros tratamientos de la alimentación realizados desde la sociología se insertan en estudios con objetivos bien distintos a la comida. En un primer momento, como nos recuerdan Mennell y sus colaboradores, tales trabajos sobre las condiciones de vida de los obreros aparecen en los estudios de Engels sobre $L a$

\footnotetext{
${ }^{7}$ En este apartado repasaremos, muy brevemente, las orientaciones teóricas que sirven de referencia a los estudiosos de la alimentación en el ámbito internacional y que podríamos considerar como clásicos en este área. En este punto es difícil establecer, como veremos, una frontera entre lo que es análisis sociológico y lo que es abordado desde la antropología.

${ }^{8}$ Fischler y Garine (1988) y Pelto (1988) en el texto sobre Carencia alimentaria, realizan sendas revisiones. El propio Fischler (1995), en su famoso estudio El (h) omnivoro, no pasa por alto una revisión comentada de las teorías dominantes en el análisis de la alimentación. La primera parte del trabajo de Godoy (1995) realiza un repaso teórico por las diferentes perspectivas. Más recientemente, el monográfico de Current Sociology, The Sociology of food: eating, diet and culture (1992), coordinado por Mennell, Murcott y van Otterlo, y el de Sociologia Ruralis (4/2002) exploran las tendencias alimentarias recientes y revisan la literatura teórica y empírica, particularmente en el ámbito anglosajón. También el estudio de Warde (1997) y la compilación de Germov y Williams (1999). En el entorno francófono, es una referencia obligada para conocer el estado de la cuestión el reciente trabajo de Poulain (2002). En España se puede ver la compilación de Contreras (1995). Gracia Arnaiz repasó los clásicos de una forma muy orientada al estudio del cambio alimentario (1997) y unos años después reseñó las investigaciones españolas que, desde la antropología y con anterioridad al año 2000, estudian la alimentación (2002). Díaz Méndez y Gómez Benito (2001) analizan, tras un repaso clásico, las líneas de investigación aún no abiertas en España en el ámbito de la sociología de la alimentación.

${ }^{9}$ En el artículo de Díaz Méndez del presente número se analiza la posición de otros clásicos en el contexto de los debates actuales sobre el cambio alimentario en las sociedades modernas.
} 
situación de la clase obrera en Inglaterra, de 1892 (1975). Más adelante se trata la alimentación al explorar la historia de la agricultura, como en los trabajos de Weber de 1909 (Weber, 1982) o en los de Durkheim sobre los comportamientos religiosos publicados en 1912 (1982). En los trabajos de Engels sobre los obreros ingleses no se presenta la alimentación como un fenómeno social en sí mismo, sino que se estudia como indicador de desigualdades. En el segundo caso, la asociación que hace Weber entre agricultura y alimentación se relaciona con los orígenes históricos de las sociedades agrarias y sus asentamientos en torno al cultivo de plantas y domesticación de animales. En el caso de los estudios de Durkheim sobre las prácticas religiosas, la alimentación, relacionada con prohibiciones y sacrificios, se integra en las relaciones sociales de las sociedades estudiadas, destacando la importancia social de las comidas en grupo y su vinculación con comportamientos de integración y relación parental. También, en otro sentido, se tratan algunos alimentos por sus cualidades sagradas y la transmisión de éstas a quienes los ingieren ${ }^{10}$.

Este tipo de aproximaciones socio-antropológicas a la alimentación comienza a cambiar con los primeros análisis sobre consumo, concretamente con Veblen, en su Teoría de la clase ociosa, publicado en 1899, y con Simmel en su Sociología de la comida escrito en 1910 e inserto en su obra El individuo y la sociedad. Para Veblen (2002) la comida, y las formas de ofrecerse y presentarse, forma parte de lo que él denomina consumo ostentoso. La alimentación, como otros consumos, es una forma de diferenciación social que utilizan las clases acomodadas para mostrar su fortaleza pecuniaria, que dice el autor (2002:75). Según Veblen, "el comienzo de una diferenciación en el consumo antecede incluso a la aparición de todo lo que pueda ser denominado propiamente fortaleza pecuniaria. Se encuentra ya en la fase inicial de la cultura depredadora... incluso antes" (2002:75). Simmel (1996) le da un tratamiento social específico a la comida, aunque inicia sus estudios enlazándolos con los ceremoniales religiosos; explora las consecuencias de la socialización de la comida y cómo la imposición de normas formales regulan las necesidades individuales de alimentos; incide en cómo se van adquiriendo pautas estéticas de comportamiento con la comida (la estilización estética) al margen del contenido alimentario.

Por su parte, Goody (1995:23-26), al referirse a los precursores de la Antropología de la Alimentación, señala también que en el siglo XIX el interés antropológico por el alimento se centró sobre todo en las cuestiones del tabú, el totemismo, el sacrificio y la comunión, es decir, los aspectos religiosos del consumo alimentario, mencionando las aportaciones de Frazer sobre alimentos

\footnotetext{
${ }^{10}$ El principio de incorporación, desarrollado en los años noventa por Fischler, ya fue considerado por Durkheim en un sentido religioso en los inicios del siglo XX.
} 
RIS

REVISTA INTERNACIONAL DE SOCIOLOGIA

№ 40, ENERO-ABRIL, 2005

CECILIA DíAZ MÉNDEZ y CRISTÓBAL GÓMEZ BENITO

permitidos y prohibidos (Frazer, 1890), las de Crawley acerca de las relaciones entre sexo y comida, o las de Smith sobre los efectos solidarios sobre la comunidad y la significación ética del comensalismo.

Más allá de estos inicios, no del todo centrados en la alimentación, pero con una aproximación social importante, aparecen ya un conjunto de autores orientados de forma específica hacia el estudio de la alimentación desde las ciencias sociales. En el repaso realizado por Poulain (2002), este sociólogo francés llega a afirmar que existen dos momentos en el estudio de la historia del pensamiento social sobre alimentación: una primera fase, que considera sin interés para la sociología, que abarca desde el nacimiento de la disciplina sociológica hasta los años 60; y una segunda fase, que se inicia con los trabajos de Levi-Straus en 1964 (Poulain, 2002:130) ${ }^{11}$.

Las funciones sociales de la alimentación han sido puestas de manifiesto desde varios puntos de vista. Una de las autoras que más ha desarrollado esta cuestión y que algunos consideran pionera en la antropología de la alimentación es Richards con su trabajo Land, labour and diet in Northern Rhodesia escrito en 1939. Considera que la búsqueda de alimentos es un acicate a la cooperación, ya que requiere de la participación del grupo para la satisfacción de sus necesidades alimentarias. Por ello se plantea el estudio de las relaciones humanas, mostrando cómo el hambre hace que se establezcan relaciones de cooperación entre los miembros de un grupo social. Estudia la producción, preparación y consumo de comida en su contexto social y cómo las maneras de comer expresan o simbolizan las pautas de relaciones sociales reforzando la cooperación dentro del grupo para buscar alimento. Dar y recibir alimentos se convierte en el símbolo de la relación dentro del grupo. En definitiva, Richards plantea que la alimentación es una actividad estructurante y organizadora de la vida social.

Desde el estructuralismo, y partiendo de Levi-Strauss, se considera que la cocina de una sociedad refleja su estructura social. Por ello, desde esta perspectiva se han dedicado a buscar las reglas que subyacen al comportamiento alimentario y su evolución a lo largo del tiempo, así como las diferencias entre distintos tipos

\footnotetext{
${ }^{11}$ En el repaso que realiza Calvo (1980) sobre la contribución de las ciencias sociales al conocimiento de la alimentación, se justifica esta apreciación de J.P. Poulain, pues los primeros trabajos de la Sociología se sitúan en los años 70. De todos modos resulta un tanto extrema la ruptura en los años 60 si consideramos la gran relevancia de los estudios sobre alimentación realizados por los antropólogos entre los años 30 y 60 . El hecho de que la sociología no haya realizado esta tarea no es motivo para negar el interés que estas aproximaciones sociales tienen para los estudios sociológicos. Algunas de las teorías desarrolladas por la antropología fueron muy criticadas en el momento de su aparición, como fue el caso, por ejemplo, de las críticas de los funcionalistas a los análisis estructuralistas de A. Richards, y fue necesario llegar a los años 80 , para recuperar estas aproximaciones teóricas y encontrar su utilidad para los análisis de la alimentación en las sociedades modernas.
} 
de sociedades en sus hábitos de presentación, preparación y elección de comidas. Lévi-Strauss, en Lo crudo y lo cocido publicado en 1964 (1968), en Le triangle culinaire, de 1965 (1965), y en El origen de las maneras de mesa, de 1968 (1997), estudia la lógica del intercambio de bienes y busca los códigos de los intercambios culinarios que acompañan las comidas cotidianas, intentándo descodificarlos (reinterpretarlos), como si se tratase de reglas lingüísticas de comunicación. Todo gira en torno a la comida y al parentesco. Para él, la cocina de una sociedad es como su lenguaje, es una forma de actividad humana universal, y no hay ninguna sociedad sin lengua y sin formas de cocinar los alimentos. Así, igual que en el lenguaje, en la cocina de una sociedad se traduce inconscientemente la estructura de esa sociedad. Esta estructura se muestra a través de las reglas culinarias y de los modelos de cocina. El modelo lingüístico de la cocina se plasma en el célebre triángulo culinario de Levi-Strauss formado por los tres vértices de la crudo, lo cocido y lo podrido.

Otros estructuralistas se han preocupado más por la variabilidad que por la universalidad, como es el caso de Douglas. Esta autora no pretende encontrar un mensaje universal, válido para toda la humanidad, codificado en el lenguaje de la comida. Por el contrario, sugiere que, cada individuo, por entrenamiento cultural, se introduce en un mundo sensorial que es pre-segmentado y juzgądo para él. Señala que, con la investigación de los hábitos culturales de la comida, podemos conocer los principios y la jerarquía de los gustos, sabores y olores, pero estos principios y jerarquías difieren de unas sociedades a otras, siendo expresiones codificadas de eventos sociales, jerarquías, exclusión e inclusión, fronteras y transacciones a través de las fronteras. Douglas (1973) afirma que las reglas de la comida sólo pueden comprenderse estructuralmente y no intentando reconstruir las relaciones de causa y efecto que pueden implicar. Revela cómo se reúnen las reglas de conducta para constituir un modelo inteligible. Todos los estructuralistas buscan códigos (la gramática que subyace a las preferencias culinarias), y buscan descifrar los significados simbólicos del acto de comer.

Barthes (1975), en su ensayo Para una psicosociología de la alimentación contemporánea también busca el código o gramática subyacente en las preferencias populares sobre la comida y lo hace a través del análisis semiológico de los anuncios y escritos de cocina.

Bourdieu (1998), es uno de los representantes actuales de este pasado estructuralista y contribuye también a estudiar la estructuración social del gusto analizando los signos de distinción social (aunque no sólo lo estudia en el caso de la comida). En su trabajo La distinción. Crítica social del sentido del gusto, se ocupa de las elecciones que hace la gente y que habitualmente son atribuidas al gusto individual. Aunque las personas eligen de acuerdo con sus preferencias, éstas son predecibles si conocemos su bagaje social. Remarca el origen social del gusto y la fuerte competencia entre grupos sociales por afirmar la distinción social. Dentro de esta perspectiva, tanto Bourdieu como Grignon han sido clasificados dentro 


\section{RIS}

de lo que se conoce como Sociología del gusto ${ }^{12}$, pues comparten la hipótesis del origen social del gusto. Esto les lleva a realizar análisis basados en las diferencias alimentarias que tienen las clases sociales, explorando los rasgos diferenciados de la alimentación burguesa y la alimentación popular actual. En definitiva, postulan que las diferencias alimentarias son diferencias de clase social y los gustos se modelan culturalmente y se controlan socialmente.

Elias (1989) ofrece unas aportaciones interesantes para explicar cómo se produce el cambio de reglas en la conducta alimentaria a través de su trabajo El proceso de la civilización publicado en alemán en 1977. Para este autor, el cambio se produce a largo plazo, y entiende que en el proceso de cambio hay aspectos que permanecen. Por ejemplo, no se han cambiado significativamente los utensilios de comida en la mesa que proceden del siglo XVIII. Sin embargo, Elias analiza la incorporación de ciertos alimentos en las dietas que se producen por apropiación de consumos, práctịcas y valores de clases sociales a través del alimento. Estudió los modales en la mesa de las clases altas de épocas diferentes $y$, aunque dice que no se trata de un cambio en una dirección única, constata que existe un comportamiento de imitación de las élites que, además de modificar los comportamientos de quienes los imitan, modifica los de los estratos que son imitados, en un proceso de diferenciación progresivo. Se cambian los modales de los estratos medios y así pierden el carácter de diferenciación de clase, lo que provoca que las élites busquen un nuevo refinamiento que los distinga de los estratos inferiores. Para Elías, los problemas del cambio alimentario requieren analizar los cambios en el proceso de la civilización, ya que la experiencia histórica aclara el significado de ciertas normas, tanto de exigencias como de prohibiciones, tanto de hábitos en la mesa como en la selección de los productos. Estudia, en definitiva, cómo se producen e interiorizan las normas alimentarias y cómo pasan de la esfera social a la esfera del sujeto.

Si como dicen Mennell, Murcott y van Otterloo (1992:8), la gran virtud del estructuralismo es que reconoce claramente que el gusto está configurado y controlado por la cultura y trata de evitar tanto el reduccionismo biológico como el etnocentrismo implícito, su debilidad radica en que se mueve hacia el polo opuesto, es decir, hacia un radical relativismo que impide cualquier posibilidad de explicar los hábitos y preferencias culinarias y alimentarias en términos de motivos, funciones o utilidades.

En otra línea teórica, aunque no desconectada totalmente de la anterior, Mennell, en su trabajo All manners of food (1985), explora cómo cada cultura gastronómica tiene normas precisas que regulan las formas de saciar el hambre y que tienen que ver con la distribución social de la comida. Se trata de

\footnotetext{
${ }^{12}$ Así los clasifica Poulain (2002).
} 
un proceso de control del apetito en situaciones de abundancia alimentaria. Este autor, que ha desarrollado la Sociología de la Alimentación en el ámbito anglosajón, utiliza algunas de las propuestas de Elías para explicar cómo los grandes cambios políticos y económicos configuran la expresión de las emociones, las maneras, el gusto y los etilos de vida de la sociedad, y los usa para estudiar los cambios en las preferencias alimentarias y en las cocinas emergentes. Compara las formas en la mesa de franceses e ingleses observando cómo, a medida que aumentan los recursos y el comercio, la división del trabajo, la pacificación interna y la formación del Estado, mejoraba la seguridad alimentaria. Así, ve posible que nuevos segmentos de la sociedad pudieran emular a las élites, y que las élites se vieran obligadas a modificar sus gustos culinarios, las maneras en la mesa y las imágenes corporales.

De un modo similar al planteado por Mennell, Goody analiza los cambios en las prácticas alimentarias desde un punto de vista histórico evolutivo y en el marco de contextos históricos y de procesos sociales más amplios, de ahí que se les llame con frecuencia desarrollistas. Goody considera que para estudiar la Sociología de la Alimentación es necesario analizar sus vínculos con los procesos de producción, distribución y consumo de alimentos, no sólo en una sociedad determinada, sino también desde una perspectiva comparada, (Goody 1995: 60 ). Cree que el análisis de la cocina tiene que relacionarse con la distribución del poder y la autoridad en la esfera doméstica, con el sistema de clase y con la estratificación y sus relaciones políticas. Explora, desde esta visión, los cambios alimentarios que se producen por efecto de la colonización y por la globalización de los sistemas de producción alimentaria.

Una aportación muy interesante es la del materialismo cultural. Su principal representante es el antropólogo Harris ${ }^{13}$. En su conocida obra Bueno para comer de 1985 considera, en oposición a Levi-Strauss, que los alimentos preferidos (buenos para comer) son aquellos que presentan una relación de coste y beneficio más favorables que los alimentos que se evitan (malos para comer), y estos costes y beneficios no son sólo de carácter nutritivo (aportes energéticos, proteínas, vitaminas o minerales), sino también de carácter práctico y ecológico (ahorro de tiempo, seguridad, accesibilidad, conservación del medio natural, etc). Toda la argumentación se presenta en oposición al estructualismo de Levi-Strauss. Harris parte de la consideración de que en todo comportamiento alimentario hay una lógica adaptativa que escapa a la conciencia del individuo (como la prohibición del consumo de cerdo entre los judíos o las vacas sagradas de la India, que tienen lógicas sanitarias o ecológicas detrás), y que no hay un origen simbólico (religioso) en estas prohibiciones, sino material. Estas prohibiciones son eficaces por sus ventajas adaptativas. Reconoce la dificultad de calcular los costes y beneficios que

${ }^{13}$ Mennell también considera a Harris desarrollista. 
están detrás de las preferencias y los rechazos alimentarios, y señala que es preciso, para conocerlos, insertar el alimento en un puzzle en el marco del sistema global de producción de alimentos y en sus consecuencias a corto y largo plazo. No olvida, sin embargo, que los alimentos, además de ser fuente de nutrición, son fuente de riqueza y poder de una minoría (Harris, 1999). Harris se opone así a la tesis de la antropología ortodoxa de que la relación entre los alimentos y sus significados es arbitraria y que no son válidas la explicaciones instrumentales de los rechazos o prohibiciones de alimentos. Entre las críticas de Fischler (1995:49) al materialismo cultural hay que señalar, según este autor, la dificultad del materialismo cultural para explicar los aspectos más sutiles y complejos de los sistemas culinarios, como, por ejemplo, las reglas de propiedad o de contextualidad intrínsecas o extrínsecas, así como que sus hipótesis son tan difíciles de probar como las del estructuralismo y que su principal dificultad reside en la noción de "adaptación óptima".

Por su parte, Mintz, en su obra Dulzura y poder, sobre el suministro y demanda de azúcar, representa otra orientación teórica dentro de la teoría general del sistema mundial, pero cuyos resultados tienen mucho en común con Harris, Goody y Mennell. Mintz critíca también al estructuralismo diciendo que el significado de los hechos sociales no lo da sólo el leerlos o descifrarlos; aunque sean resultados de aplicaciones culturales. Por el contrario, el significado resulta de la actividad. Mintz analiza el desarrollo de las plantaciones europeas de azúcar, en las Indias Occidentales y en otras partes del mundo desde comienzos del siglo XVI y la creación de un mercado de masas del azúcar especialmente en Gran Bretaña, Holanda y USA. Explica la forma en que el azúcar dejó de ser un lujo y una rareza para convertirse en la primera necesidad exótica, producida masivamente, de la clase obrera. Mintz demuestra que el incremento del consumo de azúcar sólo puede ser explicado por la combinación de factores tales como los intereses económicos, el poder político, las necesidades nutricionales y los significados culturales. La adopción del consumo de azúcar y de otros productos edulcorados por la clase obrera no fue por motivos de emulación de las élites, ni por ostentación. Mintz muestra la relevancia de los enfoques históricos, que contemplan las prácticas alimentarias en el largo plazo.

Como dicen Mennell, Murcott y van Otterloo (1992:13), la obra de Fischler (1995), El (H)omnivoro, representa el punto final del predominio del estructuralismo sobre la Sociología de la Alimentación en Francia, siendo posiblemente la apuesta sociológica más clara por los análisis de la alimentación moderna. Cuando Fischler utiliza una perspectiva de largo plazo distingue entre el comensal eterno y el comensal moderno, para manifestar tanto lo que hay de nuevo, como lo permanente en las preferencias y hábitos alimentarios de la humanidad. Reconoce las limitaciones biológicas del comensal eterno: "La situación (de abundancia de alimentos) es nueva, pero el comensal no. Sigue perteneciendo a la especie Homo Sapiens, un omnívoro cuyas características biológicas, forjadas a través de la evolución por la penuria o la incertidumbre no se ha podido aún modificar a 
pesar de las décadas de abundancia que ha vivido. El comensal del siglo XX debe hacer frente, pues, a la constante y regular abundancia actual, con un organismo más adaptado a la irregularidad de los recursos, a la incertidumbre" (Fischler, 1995:13). La paradoja del comensal moderno resulta, de un lado, de la raíz biológica de la condición de omnívoro del ser humano; la cual implica autonomía, libertad y adaptabilidad, por la diversidad de recursos comestibles a los que puede acceder en multitud de ambientes ecológicos diversos y, de otro lado, del hecho de que el ser humano no puede obtener todos los nutrientes de un solo alimento, necesitando, por tanto, un mínimo de variedad. De este modo, el omnívoro se mueve entre la diversificación, la innovación, la exploración y el cambio, y entre la precaución conservadora ante los peligros de un alimento nuevo (la neofilia y la neofobia). Además, el comensal moderno se enfrenta no a la incertidumbre del acceso al alimento, sino al de su elección, y la ansiedad está presente en esa decisión. Ambas fuerzas son motivadoras del cambio (Fischler, 1995).

Todos los autores aquí recordados han tenido críticas y revisiones de sus planteamientos, pero pueden ser tratados como clásicos en el campo de la Sociología de la Alimentación, en tanto sus trabajos aportan aproximaciones teóricas de interés para comprender los comportamientos alimentarios en las sociedades modernas. Todos ellos, y seguramente otros más no citados, pueden insertarse en los principales paradigmas de la Sociología y ser en ellos un referente. Como han puesto de manifiesto tanto Mennell, Murcott y van Otterloo como Poulain o Fischler, se muestra que el fenómeno alimentario es un tema con un gran potencial de interés sociológico, a pesar de que no haya sido un tema central en la Sociología.

Al principio, en todos los países de nuestro entorno, la Sociología de la Alimentación ha sido predominantemente empirista y ha estado centrada, básicamente, en el análisis del bienestar social y la desigualdad, asociando pobreza y alimentación. Dejando a un lado estas orientaciones, que tienen que ver más con la Sociología de la estratificación y de la desigualdad, no hay una clara y dominante orientación en la Sociología de la Alimentación. Pero cuando se incluyen los trabajos de los antropólogos, entonces se hace evidente la existencia de varias etapas teóricas, que se corresponden con las grandes fases teóricas propias de la Antropología y la Sociología generales. Tal vez no se pueda hablar de una sucesión de paradigmas, pero sí se puede hablar de una perspectiva funcionalista (representada por Richards), otra estructuralista (en la que podemos incluir, además de a Lévi-Strauss, a Douglas, o a Barthes y, en parte, también a P. Bourdieu y C. Fischler) y una rama desarrollista (cuyo representante es el materialismo cultural de Harris, pero en cuyo seno podrían situarse también Goody, Mennell y Mintz). Estas tres grandes orientaciones: funcionalismo, estructuralismo y desarrollismo, son reconocidas, aunque con leves diferencias de clasificación, tanto por Mennell, Murcott y van Otterloo (1992), como por Goody (1995) y Fischler (1995). Son formas diferentes de enfocar la alimentación, formas concretas de analizar un fenómeno alimentario común. 
Las diferencias entre estos tres paradigmas se ilustran bien comparándolos entre sí respecto a una cuestión central en la Antropología y la Sociología de la Alimentación, como es la explicación de la variabilidad de los consumos y las prácticas alimentarias (Fischler, 1995:40). Según este autor, cabe preguntarse si las normas culinarias, las prescripciones y las prohibiciones, los tabúes, etc., son fundamentalmente arbitrarios o por el contrario resultan de determinismos naturales y cumplen funciones materiales. Las respuestas sitúan a los diferentes autores a lo largo de un eje entre dos polos. En uno de ellos se encontraría la tesis de la autonomía de lo social, por la cual un hecho social sólo puede explicarse por otro hecho social. Desde este punto de vista, las reglas culinarias derivan de una especie de arbitrariedad cultural y sólo pueden comprenderse dentro de la lógica intrínseca de la cultura de la sociedad considerada. En el otro polo, se encontrarían los defensores de una sabiduría óptima de las culturas culinarias, de modo que toda particularidad alimentaria corresponde a una función adaptativa, es decir, presenta una ventaja práctica o material; así, pues, las reglas y prácticas culinarias serían una forma particularmente eficaz de adaptación cultural.

En el primer polo se encontraría el estructuralismo, y en el segundo polo el funcionalismo, pero sobre todo el materialismo cultural. Como afirma Fischler (1995:41), mientras los funcionalistas-adaptacionistas miran la comida, los estructuralistas la examinan.

\section{TEMAS Y CAMPOS DE LA SOCIOLOGÍA DE LA ALIMENTACIÓN}

La Sociología de la Alimentación que emerge desde los años ochenta presenta una triple diversidad. En primer lugar, una diversidad de perspectivas o enfoques teóricos, que constituyen diversos paradigmas explicativos de los fenómenos alimentarios, como hemos visto en el apartado anterior. En segundo lugar, una herencia multidisciplinar, comprendiendo no sólo la sociología, sino también la antropología y la historia, y nutriéndose de diversas tradiciones disciplinares que les aportan temas, perspectivas teóricas y enfoques metodológicos. Y en tercer lugar, una diversidad temática, que permite identificar sus ámbitos de actuación e interés.

McIntosh (1996:1-16) señala que desde los años ochenta el despertar del interés de los sociólogos por hacer una Sociología de la Alimentación ha ido paralelo a un interés hacia estos temas desde otras disciplinas, como la Sociología Médica, la Sociología Nutricional ${ }^{14}$, la Sociología Rural más reciente ${ }^{15}$, la antropología, la economía, la psicología y la

\footnotetext{
${ }^{14} \mathrm{McIntosh}$ distingue entre una sociología en la nutrición y una sociología de la nutrición. La primera sería una especie de epidemiología social de los problemas (médicos) de la nutrición, es decir, el análisis de las variables sociales que afectan a la salud en relación con la comida. La segunda se ocuparía de las actividades sociales relacionadas con la dietética y la nutrición (McIntosh, 1996:11-12).

${ }^{15}$ Que se ha ocupado de los problemas de la globalización de los sistemas agroalimentarios, la concentración de la agroindustria, la mercantilización de la alimentación, la calidad y la seguridad alimentaria o las respuestas de los consumidores a estos productos.
} 
ciencia de la nutrición. Aún así, este autor reclama el papel independiente de una Sociología de la Alimentación y de la Nutrición. Por su parte, Mennell, Murcott y van Otterloo (1992), también tienen en cuenta la investigación antropológica, histórica y socio-biológica, además de la específicamente sociológica. Se puede decir que la Sociología de la Alimentación es deudora, o se nutre, de la Sociología del Consumo, de la Sociología Rural y de los Sistemas Agroalimentarios, de la Sociología de la Cultura, de la Antropología Social y Cultural, de la Sociología de la Salud y del Cuerpo, de la Historia de la Alimentación y de la Ciencia de la Nutrición. Esta diversidad de herencias revela la condición de la alimentación como hecho social total ${ }^{16}$.

Por último, presenta también una gran diversidad temática. A falta de una definición expresa del objeto de la Sociología de la Alimentación, ésta se puede identificar por los temas de los que se ocupa y por la delimitación del campo temático, aunque este campo tampoco haya sido expresamente acotado.

Para ofrecer un cierto panorama de los campos y temas de los que se ocupa la Sociología de la Alimentación, hemos analizado el contenido de tres libros de referencia que creemos relevantes en el análisis de la alimentación desde una perspectiva social y que constituyen la constatación en el entorno europeo de una disciplina aún inexistente en España. El primero trata de la revisión del estado de la cuestión realizada por Mennell, Murcott y van Otterloo en 1992 para la revista Current Sociology. El segundo es el trabajo del francés Poulain editado en 2002 (Sociologies de l'alimentation), que se ha convertido también en referencia para la Sociología de la Alimentación, ampliando la aportación francesa y siendo más actual que el anterior. El tercero es ya un clásico para los estudiosos de la alimentación, se trata de la obra de McIntosh (1996): Sociologies of Food and Nutrition. Los títulos ya revelan un primer campo: la comida, los alimentos y el hecho de comer, la dieta y la nutrición, y la cultura ${ }^{17}$.

La descripción del campo que hacen Mennell, Murcott y A. Otterloo resulta de una suma de orientaciones y nos permite ver la enorme variedad de temas, enfoques e intereses de los estudios socio-antropológicos sobre la alimentación, pero no hay una propuesta de formalización de esta subdisciplina, ni una definición expresa de su objeto. Estos autores subrayan que la comensalidad está en el centro de la sociabilidad, por lo que es natural que la Sociología de la Alimentación conecte con los temas clave de la teoría sociológica. Por esto mismo, es una disciplina

\footnotetext{
${ }^{16}$ Así lo sostiene Poulain usando a Mauss.

17 Otros libros se refieren al gusto, aunque en muchos casos resulta difícil la diferenciación temática. Así , por ejemplo, el conocido libro de Fischler, El (H)omnivoro (1995) lleva por subtítulo El gusto, la cocina y el cuerpo. La compilación de Germov y Williams (1999) lleva como subtítulo The social appetite, tras un título en el que puntualiza Sociology of food and nutrition. Vease también el libro de Warde (1957).
} 
poco unificada y muy conectada con otras disciplinas afines. El desarrollo de la clara multidisciplinariedad de la alimentación parece la propuesta de estos autores.

Poulain se centra en los procesos que están configurando el sistema alimentario de las sociedades actuales, especialmente en las sociedades opulentas occidentales, constatando la relevancia sociológica del estudio del fenómeno alimentario para comprender aspectos fundamentales de las sociedades actuales. Además, intenta definir el campo y situarlo epistemológicamente en la tradición sociológica, realizando una interesante revisión de autores y paradigmas de las ciencias sociales. Hace un intento de definición de la socio-antropología de la alimentación y considera que hay un espacio social alimentario susceptible de análisis específico, aun dentro de la multidimensionalidad de la alimentación (o incluso precisamente por ella).

McIntosh realiza una revisión de las aproximaciones sociológicas al estudio de la alimentación como los autores anteriores, pero, como novedad, la realiza también de la nutrición, aproximándose a la Sociología Médica y a la Sociología Nutricional, por un lado, y la Sociología Rural, por otro. Reclama el papel independiente de una Sociología de la Alimentación y de la Nutrición.

A fin de comprender estas posturas que no son del todo discrepantes, pero tampoco coincidentes, vamos a comentar brevemente las aproximaciones realizadas en esos tres manuales a través de los temas tratados en ellos ${ }^{18}$. El trabajo de Mennell y sus colaboradores puede agruparse en varios apartados.

- El desarrollo de las culturas culinarias. Aquí se explora, en general, el mundo de las cocinas, que parece haber sido uno de los temas centrales de la Sociología de la Alimentación, especialmente en la tradición etnológica o antropológica, pero también en la historia de las mentalidades.

- Las tendencias nutricionales actuales. Estas tendencias se sustentan en los estudios empíricos sobre los estados nutricionales de las poblaciones y su evolución, que tienen ya una larga historia en muchos países y que han sido estudios pioneros sobre la alimentación. Dentro de este grupo hay que incluir también los estudios que se han ocupado del desarrollo de la ciencia de la nutrición y de otras disciplinas afines, pero también los más recientes sobre el consumo y los hábitos alimentarios en las sociedades actuales (compartiendo el interés con economistas y psicólogos), especialmente en función de las variables sociodemográficas básicas (edad, sexo y clase social), así como sobre las tendencias en las políticas nutricionales.

- Las creencias y prácticas sobre la comida y la salud. Se trata de otro subcampo que ha sido muy atendido desde la Antropología Social y Cultural y que relaciona alimentación y salud.

- Los desórdenes en la comida. Estudios muy ligado a los modelos de

\footnotetext{
${ }^{18}$ En los tres libros comentados se sigue, en cierta medida, el propio índice de los manuales, realizando comentarios sobre su contenido.
} 
consumo de las sociedades opulentas y a los trastornos alimentarios actuales como la bulimia y la anorexia; se exploran aquí también sus vínculos con el género.

- La carencia y la saciedad. Un tema que entronca con algunos de los estudios de los clásicos y que abarca tanto el presente como el pasado y tanto a las sociedades opulentas (viejas y nuevas formas de privación alimentaria en las sociedades occidentales contemporáneas) como a las más pobres y desnutridas. Todo ello constituye una forma de estudiar la desigualdad social y sus relaciones con la alimentación, pero no sólo eso, también se han interesado por las prácticas de ayuno y por las del banquete vinculadas a estilos de vida, orientaciones religiosas o filosóficas, etc.

- La tecnología alimentaria y su impacto. Un tema muy relevante en el estudio de los sistemas alimentarios modernos y sus transformaciones; entre ellas, los efectos sobre la alimentación de la industrialización de la agricultura, de la producción artesana de alimentos, de los cambios en la distribución, preparación y consumo de alimentos. Se estudia también el desarrollo de la tecnología alimentaria y sus implicaciones para el consumo.

- El impacto de culturas dominantes. La tradiciones culinarias (productos y comidas) se han visto alteradas por el trasiego de comestibles y comidas ya desde la época de los imperios coloniales y sus herencias (tanto en las metrópolis como en las colonias). Así mismo, se han estudiado los efectos de la industrialización y de la construcción del imperio en los comportamientos y hábitos alimentarios de las colonias. Todos estos procesos de intercambios de productos y comidas se intensifican hoy con las migraciones, dando lugar al auge de las cocinas de los inmigrantes y la apertura hacia los estudios alimentarios asociados a la identidad.

- El espacio público de la comida. El estudio sobre los espacios públicos de la comida se ha ido ampliando en los últimos años. Se trata aquí del desarrollo de las cocinas profesionales y de la comida fuera del hogar, tanto desde el punto de vista del desarrollo de la gastronomía profesional, como de la restauración alternativa o complementaria a la comida del hogar. Se exploran también los espacios de la alimentación institucional (colegios, hospitales, cárceles, cuarteles, centros de trabajo, conventos, etc.).

- El espacio privado de la comida (o la cocina y la economía doméstica). Es el otro espacio de la cocina y se ponen de manifiesto sus múltiples y recientes transformaciones. Un tema novedoso de estudio es el de la formación, más o menos formal, en materia culinaria, especialmente de las mujeres. Con ello, se atiende al papel de la mujer contemporánea en la cocina y en la familia, la democratización y la influencia de la economía y de la tecnología doméstica, los efectos de la comida industrial y la comida fuera de casa sobre la cocina doméstica. Otros temas cuentan con una mayor tradición, como las relaciones entre la alimentación y la división del trabajo en la casa o las desigualdades en el reparto 


\section{RIS}

de alimentos en el hogar. Con perspectiva de género se ha avanzado en esta línea de investigación.

Frente a este panorama descriptivo, y en cierto modo de carácter enciclopédico, del trabajo de Mennell, Murcott y van Otterloo, la obra de Poulain aborda el campo de la Sociología de la Alimentación de una forma más integrada y articulada, en torno al hilo conductor de los cambios sociales y sus efectos sobre la alimentación. Se puede afirmar que el texto de Poulain es un análisis sobre la modernidad alimentaria por varias razones: en primer lugar, por aportar un análisis centrado en las tendencias y los problemas alimentarios actuales; en segundo lugar, por apoyarse en datos empíricos recientes sobre el cambio alimentario; en tercer lugar, por encontrarse todo ello arropado por un relevante análisis teórico sobre el fenómenos de la alimentación en las sociedades contemporáneas.

La parte referida a los asuntos propios de la Sociología de la Alimentación ${ }^{19}$ ocupa la primera parte del libro de Poulain. La segunda parte la dedica íntegramente a la presentación de las perspectivas de análisis que ofrecen claves interpretativas de los fenómenos alimentarios, ofreciendo una panorámica sobre los paradigmas sociológicos y la forma en que los diferentes autores exploran la alimentación. Tras una revisión de estos paradigmas asociados al estudio de los comportamientos alimentarios, analiza las posiciones de los autores actuales que han explorado, y exploran, el hecho alimentario con una perspectiva próxima a la sociología ${ }^{20}$. Pero, como indicábamos, es la primera parte la que dedica a la exposición temática, destapando con ello aquellas áreas de trabajo consideradas propias de la investigación social en el terreno de la alimentación. Cuenta con un importante soporte empírico y realiza un análisis de aquellas investigaciones que sustentan las hipótesis que va desarrollando a lo largo del texto. El análisis temático se sustenta en varios campos:

- Los efectos de la globalización y la relocalización. Explora los efectos contrapuestos generados, por un lado, por la globalización y sus tendencias homogeneizantes, $y$, por otro, por la alimentación local que retorna a los particularismos identitarios. Todo ello entronca con las identidades alimentarias y el exotismo y la variedad aportada por las culturas.

- El consumo doméstico y sus transformaciones. Se exploran aquí dos tipos de debates. Por una parte, los efectos de la industrialización de la alimentación sobre

\footnotetext{
${ }^{19}$ El propio Poulain afirma que es necesario utilizar este término en plural, pues no hay una sola Sociología de la Alimentación, sino diversas aproximaciones teóricas y metodológicas.

${ }^{20}$ Poulain realiza un análisis de las sociologías de la alimentación relacionando posiciones epistemológicas y autores. Sitúa a éstos en un doble eje: por un lado, de acuerdo con su posición teórica respecto a la relevancia de lo social; por otro, según se acerquen más o menos a una visión conservadora de los cambios o a una visión más dinámica (2002:190). En el esquema sólo se reseñan autores anglosajones y franceses de acuerdo con las perspectivas planteadas por el propio Poulain.
} 
el consumo doméstico. Por otro lado, las transformaciones de la organización de la vida cotidiana y sus efectos sobre las formas de comer. Se analiza, por ejemplo, el papel de la restauración fuera del hogar y el retorno a la comida casera; las oscilaciones $u$ oposiciones entre la cocina empaquetada (industrializada) y la cocina de placer; el papel de la restauración fuera del hogar; la figura del comensal; el sistema de restauración y la decisión, el retraimiento y el retorno de lo doméstico.

- La norma y la anomia alimentaria. En un tercer apartado el autor explora los cambios más característicos en torno a la tesis de la gastro-anomia y los debates asociados a ella: el debilitamiento de los controles sociales y la multiplicación de los discursos alimentarios, la relevancia de las clases sociales para comprender los comportamientos alimentarios y sus pautas normativas, o las relaciones entre las normas y las prácticas, con una teoría propia del autor desarrollada con un importante soporte empírico.

- Los riesgos y las elecciones alimentarias. Este campo constituye uno de los más novedosos dentro de la nueva Sociología de la Alimentación y ha servido para conectar estos estudios con aspectos centrales de la Sociología en las sociedades actuales, como el enfoque de la sociedad del riesgo. Muestra con diferentes enfoques las formas en que se ha abordado el riesgo alimentario. Cuestiones como el malentendido de la calidad, el riesgo y las sociedades modernas, el riesgo de los expertos y el riesgo de los profanos, el riesgo como una constante de la alimentación humana, o la gestión democrática del riesgo y la reconstrucción social de los alimentos, muestran la forma en que se han abordado estos temas.

- La salud y la medicalización de la alimentación. Establece una relación entre modernidad alimentaria y salud relacionando así el campo de análisis social con el nutricional. En esta línea se explora también la obesidad, para ilustrar las transformaciones en las formas de pensar sobre cuestiones alimentarias y de salud en las sociedades actuales, donde las fronteras entre ambas son difusas.

Por su parte, la obra de McIntosh analiza las relaciones entre cultura y alimentación desde diversos marcos teóricos: las aproximaciones sociológicas a la cultura, la teoría de la modernización, las teorías de la modernidad avanzada, la teoría crítica y el postmodernismo, la sociología del riesgo, la nutrición y la salud, los nuevos movimientos sociales, etc. El interés de este libro radica, entre otros aspectos, en el intento del autor de relacionar los temas alimentarios con la teoría sociológica general ${ }^{21}$. McIntosh presenta varios grandes campos de estudio y en cada uno de ellos conecta diversos temas con las teorías sociológicas generales.

\footnotetext{
${ }^{21}$ Esta visión es particularmente interesante si consideramos que el vínculo que establece Poulain se centra más en la relación entre autores y paradigmas teóricos, y no tanto entre aquellos y las teorías sociológicas contemporánea (con la excepción, probablemente, de la teoría del riesgo). Sirve así como interesante complemento del anterior. Bien es cierto que Poulain desarrolla un apartado sobre las dimensiones del espacio social alimentario (capítulo 6) que puede ser utilizado, y así lo plantea, para el estudio de los modelos alimentarios (Poulain, 2002: 228).
} 
- La organización social de las actividades alimentarias y el estatus nutricional. Establece una relación entre las actividades alimentarias y las teorías de la familia, explorando el papel de la mujer en la organización social de la producción, preparación y el consumo alimentarios.

- La estratificación social o la distribución de la alimentación y la nutrición. Considera las teorías de la estratificación social y el desarrollo en la solución de los problemas alimentarios.

- El cuerpo y la sociología. En contacto con otras disciplinas, como la medicina, establece vínculos entre la salud y la sociología del cuerpo (las dimensiones simbólicas y la construcción social del cuerpo).

- La sociología del hambre. Insiste en la necesidad de no perder de vista los países con carencias alimentarias y plantea el análisis sobre las causas y las consecuencias del hambre.

- La alimentación y el cambio social. Desarrolla las aplicaciones de las teorías del cambio social al cambio alimentario.

- El Estado, la alimentación y la nutrición. Constata que hay pocos campos en los que los comportamientos se encuentren tan regulados, a través de políticas nutricionales y sanitarias. Resalta el efecto que esto tiene sobre toda la cadena agroalimentaria. Apunta además hacia un campo nuevo de análisis relacionado con los derechos ciudadanos en referencia a la protección alimentaria.

- La alimentación, la nutrición y los problemas sociales. Analiza la alimentación como problema social, abriendo así nuevas vías de análisis que deben afrontar las teorías clásicas y actuales de la sociología, desde la teoría marxista al constructivismo.

Este repaso por los tres textos citados nos permite afirmar que existe un objetivo común: comprender la alimentación desde un punto de vista social para afrontar el estudio del cambio alimentario en las sociedades actuales. Aunque se trate de un objetivo amplio y de difícil delimitación, los tres textos analizados justifican la relevancia de al menos cuatro campos de análisis.

Un primer campo hace referencia a los efectos de los cambios macrosociales sobre la alimentación. Quizás como punto de referencia haya que considerar aquí el efecto de la globalización o mundialización y sus interrelaciones con las culturas alimentarias locales. En este análisis pueden incluirse además las explicaciones sobre la construcción de modelos alimentarios, así como el impacto de los modelos dominantes y la resistencia de los minoritarios. Dentro de este campo se pueden incluir también las aportaciones sobre los sistemas agroalimentarios procedentes de la sociología agroalimentaria.

Un segundo campo estaría formado por aquellos temas asociados a las modificaciones microsociales de la alimentación contemporánea. Nos referimos aquí a todas aquellas referencias al cambio alimentario dentro del hogar, así como a su extensión pública (restauración privada e institucional). La compra, la cocina, la dieta, las preferencias alimentarias, el gusto; un gran número de términos y de espacios de relación relativamente novedosos en los análisis sociológicos. 
Un tercer campo haría referencia a las cuestiones de carácter nutricional en un sentido general y asociado a la estratificación social. Se tratan aquí los estados de salud de la población y su relación con los factores sociales que los modifican. Siempre presente, el tema de las desigualdades y la consideración de la alimentación como problema social. En este mismo área, las creencias sobre comida y salud conforman el sustrato sobre el que se asientan los análisis que unen salud y alimentación.

Un cuarto y último campo entraría en el ámbito de la producción alimentaria, aunque bien es cierto que no ha sido tratado en toda su extensión. Aquí estarían incluidos los estudios sobre los cambios en las tecnologías agroalimentarias o todas aquellas cuestiones asociadas al riesgo alimentario. Aunque se han tratado en relación con las nuevas situaciones de riesgo y seguridad alimentaria asociadas a la modernidad, en la mayor parte de los casos las exploraciones hacia el campo de la producción han sido menores, tanto hacia el sector primario (la agricultura) como al secundario (la industria).

Otros dos campos han sido menos atendidos: el de las políticas alimentarias y el de los efectos ecológicos de los sistemas alimentarios. En cuanto al primero, si bien ha estado vinculado a los temas de la producción, ahora se relaciona sobre todo con los campos del consumo. En este sentido, se está prestando atención al marco institucional de la regulación de la seguridad alimentaria (las Agencias de Seguridad Alimentaria y el ordenamiento jurídico).

En cuanto al segundo se podría calificar de socio-ecología de la alimentación humana. La socio-ecología de la alimentación humana es un campo incipiente de gran importancia que no se limita a los estudios sobre la seguridad y el riesgo alimentario, tan presentes en la sociología de la sociedad del riesgo y en la sociología del consumo, sino que aborda las implicaciones ecológicas de toda la cadena agroalimentaria en cada sistema alimentario: por ejemplo, la cuestión de los residuos, que permitiría abordar la alimentación desde la perspectiva del metabolismo social ${ }^{22}$ y sus implicaciones ecológicas. En este sentido, el libro de Rifkin (2001), sobre la cultura de la carne en las sociedades opulentas actuales, es un ejemplo (entre otros muchos sobre otros productos) de análisis que integra la perspectiva histórica y sociológica y las relaciones entre la industria agroalimentaria (la producción), el consumo y la cultura, para mostrarnos sus (devastadoras) implicaciones ecológicas o el de Lappé (1982) ${ }^{23}$.

\footnotetext{
${ }^{22}$ Utilizamos el concepto de metabolismo social según la acepción de Fischer-Kowalsky (2002), como el flujo de materia y energía en la sociedad a través de la cadena de extracción, producción, consumo y eliminación.

${ }^{23}$ Otro interesante análisis en esta línea es el del periodista Schlosser sobre el sistema de Fast Food. Otra línea de interés es la del análisis energético de los sistemas de producción de alimentos, como los de Pimentel y Pimentel (1979). Un análisis del impacto ambiental del consumo doméstico aplicado al País Valenciano puede verse en García (1997), en el que, entre otros aspectos, considera el consumo alimentario.
} 


\section{RIS}

Como ya hemos adelantado, es en la obra de Poulain donde podemos encontrar un cierto intento explícito de definir el objeto de una Sociología de la Alimentación. Este sería el análisis de lo que él denomina espacio social alimentario ${ }^{24}$, concebido como una herramienta para el estudio de los modelos alimentarios, un espacio social alimentario delimitado doblemente por las constricciones biológicas de la fisiología humana y por las constricciones ecológicas del medio, y que presenta la siguientes dimensiones: el espacio "comestible", el sistema alimentario, el espacio culinario, el espacio de los hábitos de consumo, la temporalidad alimentaria y el espacio de la diferenciación social. Estas dimensiones son de alguna forma los grandes campos de la socio-antropología de la alimentación, junto a la construcción social de los alimentos.

En Poulain (2002:243) se define el objeto de la socio-antropología de la alimentación como "la manera en que las culturas y las sociedades delimitan y organizan el espacio de libertad dejado por el funcionamiento fisiológico del sistema digestivo del hombre y por las modalidades de explotación de los recursos puestos a disposición por el medio natural o susceptibles de ser producidos en el marco de las restricciones biofísicas y climatológicas del biotopo", y más adelante afirma "es la originalidad de la conexión bio-antropológica de un grupo humano y su medio lo que constituye el objeto de la socio-antropología de la alimentación"(2002:244). Desde este punto de vista, la socio-antropología de la alimentación se interesa por las interacciones entre lo biológico, lo ecológico y lo social, y ahí radica su originalidad, convirtiéndose no sólo en un fenómeno social total, sino también en un fenómeno humano total. ${ }^{25}$

En consecuencia, según Poulain, los retos de la socio-antropología de la alimentación son de dos tipos. Primero, por su anclaje epistemológico sobre el "espacio social alimentario", que simultáneamente la inscribe en las ciencias sociales y la abre a la interdisciplinaridad, le permite investigar las relaciones entre lo cultural y lo fisiológico, por una parte, y entre lo cultural y el medio natural, por otra. Segundo, por su dimensión estructurante de la organización social, el espacio social alimentario ocupa una posición transversal en las ciencias sociales y humanas y crea la condición de una metabolización del saber socio-antropológico; un espacio de frontera para medir las interacciones entre lo biológico y lo cultural.

De este modo, las dos aproximaciones sociológicas al fenómeno de la alimentación que distingue Poulain son necesarias (2002:244), a saber : la que, respetando el principio de la autonomía de lo social, se interesa por las dimensiones social-

\footnotetext{
${ }^{24}$ Plantea este término en referencia a Condominas y apuesta por su pertinencia, pues, por un lado, permite avanzar en el eterno dilema entre el determinismo cultural y el material, y a la vez crea las condiciones para establecer un vinculo entre el hombre y la naturaleza (Poulain, 2002:222).

${ }^{25}$ Poulain hace aquí referencia en el primer término a Mauss y en el segundo a Morin. Se puede ver su análisis en Poulain ( 2002: 243-244).
} 
mente impuestas del hecho alimentario; y la que, considerando a la alimentación como un hecho social total, se abre al diálogo con otras ciencias, como las de la nutrición y la epidemiología, cuestionando la brecha epistemológica sobre la que se basa la Sociología.

\section{CONCLUSIONES}

En este artículo hemos pretendido, a modo de introducción de este número monográfico, definir someramente el contexto de sociogénesis de la Sociología de la Alimentación. Este contexto se vislumbra al aproximarse a varios aspectos: a la diversidad de su herencia académica, a sus estrechas relaciones con otras disciplinas afines, a la diversidad de sus orientaciones teóricas y a la gran variedad de temas que entroncan con casi todos los grandes campos de la sociología. La sociología está presente a través de la familia y la estructura social, del trabajo y la religión, del cambio social y la modernización, de la etnicidad y la diferenciación social, del poder y la desigualdad, de la sociabilidad y el conflicto, de la política y el Estado, de la economía y el cambio tecnológico, de la producción y el consumo.

La Sociología de la Alimentación está lejos de ser una subdisciplina unificada y hay serias dudas de que pueda serlo algún día por su carácter transversal, pero el desarrollo de las últimas décadas apunta a una progresiva convergencia de temas y preocupaciones y a una progresiva articulación de enfoques y metodologías. Quizás esto sea suficiente para pensar en un desarrollo propio también en España.

En la obra de los sociólogos clásicos se ha prestado atención preferentemente a los aspectos productivos, usando la alimentación como medio para conocer otras manifestaciones sociales: la desigualdad, el poder, la religión, etc. Después, las orientaciones sociológicas hacia el mundo del consumo le otorgaron un carácter más general, aunque supeditado al ámbito del análisis del mercado. Tanto es así que la Sociología de la Alimentación se ha identificado muchas veces con una sociología del consumo alimentario. Paralelamente y sin apenas conexión con la sociología del consumo, se estaba desarrollando una sociología de los sistemas alimentarios procedente de la economía y de la sociología agraria, en particular de los agro-food studies; una línea que ha estado centrada sobre todo en la producción, pero que se ha ido acercando al mundo del consumo ${ }^{26}$. Uno de los retos actuales de la Sociología de la Alimentación es articular ambos aspectos, producción y consumo, en unos mismos marcos teóricos.

\footnotetext{
${ }^{26}$ En la revista Sociologia Ruralis se ha venido publicando desde 2002 un debate en torno a este tema. Véase especialmente Goodman (2002), Goodman and Du Puis (2002), Lockie (2002), Fine (2004) y la totalidad del vol. 42/4, de octubre de 2002.
} 


\section{REFERENCIAS BIBLIOGRÁFICAS}

BARTHES, R (1975), "Pour une psychosociologie de l'alimentation contemporaine", en Brillat Savarin (ed.), Physiologie du gồt, Hermann, Collection Savoir, Paris.

BOURDIEU, P. (1998), La distinción. Criterio y bases sociales del gusto, Madrid, Taurus.

CALVO, M. (1980), "De la contribution actuelle des sciences sociales et humaines á la connaissance de l'alimentation", Ethnologie francaise, X, (3), pp. 335-352.

CONTRERAS, J. (1995), Alimentación y cultura. Necesidades, gustos y costumbres, Barcelona, Universitat de Barcelona.

DOUGLAS, M. (1973), Pureza y peligro: un análisis de los conceptos de contaminación y tabú, Madrid, SigloXXI.

DURKHEIM, E. (1998), Las reglas del método sociológico y otros escritos sobre filosofia de las ciencias sociales, Madrid, Alianza.

(1982), Las formas elementales de la vida religiosa, Madrid, Akal.

DÍAZ MÉNDEZ, C. y C. GÓMEZ BENITO, (2001), "Del consumo alimentario a la Sociología de la Alimentación", Distribución y Consumo, n 60 , pp. 5-23.

ELIAS, N. (1989), El proceso de la civilización, Madrid, Fondo de Cultura Económica.

ENGELS, F. (1975), "Prefacio a la situación de la clase obrera en Inglaterra", en C. Marx y F. Engels, Obras Escogidas, Madrid, Editorial Fundamentos.

FINE B. (2004), "Debating production.consumption linkage in food studies", Sociologia Ruralis, Vol 4 (3), pp.332-342.

FISHLER C. e I. GARINE (1988), "Ciencias humanas y alimentación: Tendencias actuales de la investigación europea”, en Carencia Alimentaria en una perspectiva sociológica, Barcelona, Serval, pp.65-87.

FISCHER-KOWALSKI, M. (2002), "El metabolismo de la sociedad: sobre la infancia y adolescencia de una naciente estrella conceptual", en M. Redclift y G. Woodgate, Sociologia del medio ambiente. Una perspectiva internacional, Madrid, McGraw Hill.

FISCHLER, C. (1995), El (h)omnivoro, El gusto, la cocina y el cuerpo, Barcelona, Anagrama.

FRAZER, J. (1984), La rama dorada 1984, Madrid, FCE.

GARCÍA, E. (1997), "El impacto ambiental del consumo doméstico: un análisis socioecológico", comunicación presentada a la Conferencia sobre Sociología y Medio Ambiente, Pamplona, noviembre de 1997.

GERMON, J. y L.A. WILLIANS (1999), Sociology of food and nutrition. The social appetite, Oxford, Oxford University Press. 
GOODMAN, D. (2002), "Rethinking food production-consumption: integrative perspectives", Sociologia Ruralis, vol. 42 (4), pp. 271-277.

GOODMAN, D. y M. DUPUIS (2002), "Knowing Food and Growing Food: Beyond the Production. Consumption Debate in the Sociology of Agriculture", Sociologia Ruralis, vol 42, No 1, Enero 2002, pp. 5-22.

GOODY, J. (1995), Cocina, cuisine y clase, Barcelona, Gedisa.

GRACIAARNAIZ, M. I.(1997), La transformación de la cultura alimentaria. Cambios y permanencias en un contexto urbano, Madrid, Ministerio de Educación y Cultura.

(2002), "La alimentación en el umbral del siglo XXI: una agenda para la investigación sociocultural en España", en Gracia Arnaiz (comp), Somos lo que comemos, Barcelona, Ariel, pp.16-38.

HARRIS, M. (1999), Bueno para comer, Madrid, Alianza.

LAMO DE ESPINOSA, E. (1996), Sociedades de cultura, sociedades de ciencia, Oviedo, Ediciones Nobel.

LAPPÉ, F. M. (1982), Diet for a small planet, Nueva York, Ballantine.

LEVI -STRAUSS, C. (1965), "Le triangle culinaire", L'Arc, n² 26, pp.19- 29.

(1968), Lo crudo y lo cocido, Mitológicas I, México, Fondo de Cultura Económica.

(1997), El origen del la maneras de mesa, Mitológicas III, México, Siglo XXI.

LOOCKIE, S. (2002), "The invisible mouth: movilizing the consumer in food production-comsumption networks", Sociologia Ruralis, vol. 42 (4), pp. 278-294.

MCINTOSH, WM.A. (1996), Sociology of food and nutrition, Nueva York, Plenium Press.

MENNELL, S. (1985), All Manners of Food. Eating and Taste in England and France from the Middle Ages to the Present, Londres, Basil Blackwell.

MENNELL, S., A. MURCOTT, y A. van OTTERLOO (1992), "The Sociology of Food: Eating, Diet and Culture", Current Sociology, Vol. 40 (2).

MINTZ, S. W. (1985), Sweetness and Power: The place of sugar in Modern History, Nueva York, Viking Penguin nc.

PELTO, H. (1988), "Tendencias de la investigación en la antropología nutricional”, Carencia alimentaria en una perspectiva sociológica, Barcelona, Serval, pp.45-64. 87.

PIMENTEL, D. y M. PIMENTEL (1979), “Food, energy and society”, Nueva York, Wiley.

POULAIN, J. P. (2002), Sociologies de l'alimentation, París, Presses Universitaires de France.

RICHARDS, A. (1939), Land, Labour and Diet in Northem Rhodenia, Londres, Oxford University Press. 


\section{RIS}

REVISTA INTERNACIONAL DE SOCIOLOCIA

№ 40, ENERO-ABRIL, 2005

RIFKIN, J. (2001), Ecocidio. Ascesa e caduta della cultura della carne, Milán, Mondadori.

SCHLOSSER, E. (2002), Fast Fodd. El lado oscuro de la comida rápida, Barcelona, Gijalbo.

SEN, A. (1981), Poverty and famines: An essay on entitlement and deprivation, Nueva York, Oxford University Press.

SIMMEL (1986), El individuo y la sociedad, Barcelona, Península.

VV.AA. (2002), Sociologia Ruralis, vol.42 (4).

VEBLEN, T. (2002), Teoria de la Clase Ociosa, Madrid, Fondo de Cultura Económica.

WARDE, A. (1997), Consumption, Food and Taste, Londres, Sage Publications.

WEBER, M. (1982), Historia agraria romana, Madrid, Akal. 